\title{
Microwave treatment in oil refining
}

\author{
Adrian Porch • Dan Slocombe · Jan Beutler • Peter Edwards • Afrah Aldawsari • \\ Tiancun Xiao • Vladimir Kuznetsov $\cdot$ Hamid Almegren $\cdot$ Saud Aldrees • \\ Naif Almaqati
}

Received: 28 June 2012/ Accepted: 31 July 2012/Published online: 18 August 2012

(C) The Author(s) 2012. This article is published with open access at Springerlink.com

\begin{abstract}
In this paper, we discuss the potential of using microwave techniques in the refinement of heavy fraction of petroleums such as bunker oil. After discussing the fundamental issues associated with conversion of microwave energy into heat, we present measurements of the dielectric properties of heavy oils at $2.45 \mathrm{GHz}$ using a highly sensitive resonant cavity method, and also over a broader frequency range $(100 \mathrm{MHz}$ to $8 \mathrm{GHz})$ using a coaxial probe technique. We find that the dielectric loss is very small even in these heavy oils, but still may be sufficiently large to provide efficient conversion of microwave energy into heat on untreated samples, and could be massively enhanced by means of a microwave-absorbing additive (e.g., carbon black). We conclude by discussing the design of a suitable microwave actuator for heavy oil cracking within a flow process.
\end{abstract}

Keywords Microwave $\cdot$ Dielectric $\cdot$ Petroleum

A. Porch $(\bowtie) \cdot$ D. Slocombe $\cdot$ J. Beutler

School of Engineering,

Cardiff University, Cardiff CF24 3AA, UK

e-mail: PorchA@cf.ac.uk

P. Edwards - A. Aldawsari - T. Xiao - V. Kuznetsov ·

S. Aldrees · N. Almaqati

Inorganic Chemistry Laboratory, Oxford University,

Oxford OX1 3QR, UK

H. Almegren - S. Aldrees - N. Almaqati

Petrochemical Research Institute, King Abdulaziz City for

Science and Technology, P.O. Box 6086, Riyadh 11442,

Kingdom of Saudi Arabia

\section{Introduction}

There is an ever-increasing global demand for high value, light olefins such as ethylene, propylene and butylene, which form the essential building blocks for the petrochemical industry. However, these demands, coupled with more stringent environmental regulations for automotive fuel, cannot be met by conventional petrochemical technologies.

Here, we investigate the use of microwave technologies to assist in the high-efficiency conversion of crude oil to light olefins, aromatics and other high-value chemicals. Almost all of the targeted chemical reactions involving the breaking of $\mathrm{C}-\mathrm{C}$ bonds are highly endothermic in nature, requiring considerable inputs of energy. It might be possible to heat the crude oil directly with very high power microwaves to attain the required reaction temperatures. However, this can be aided by the addition of high surface area, microwave-absorbing catalysts or additives (such as methanol or water, or solids such as carbon black), which provide a means for the selective heating of the additive within the crude oil emulsion. Either way, we expect the application of microwaves to assist in the breaking of $\mathrm{C}-\mathrm{C}$ bonds from fractions of the whole crude oil to generate valuable chemicals and premier fuel products.

In this paper, we will first discuss the main issues affecting the efficient heating of materials using microwaves, then present microwave characterization of crude oil samples, and finally discuss the design of a suitable actuator for direct or assisted heating of these samples.

\section{Literature survey}

Microwaves are electromagnetic waves whose frequencies lie in the range between about 300 and $30 \mathrm{GHz}$, 
corresponding to free space wavelengths from about $1 \mathrm{~m}$ down to $1 \mathrm{~cm}$, respectively. Beyond $30 \mathrm{GHz}$, the microwave spectrum gives way to the millimeter ( $\mathrm{mm}$ ) wave spectrum, and below $300 \mathrm{MHz}$ the radio frequency (RF) spectrum. Microwave heating is now a standard processing tool for the industrial chemist [1-4].

In the microwave part of the electromagnetic spectrum, heating is normally a result of the conversion of the electric field energy part of the microwave signal, generally termed "dielectric heating". The efficiency of this process is determined by the dielectric loss (i.e. loss tangent) of the material. This, in turn, is related to the polar nature of the material and high conversion efficiency is found in highly polar materials (e.g., water) or those of moderate electrical conductivity (e.g., carbon black, of conductivities around 0.1-1 S/m around $900 \mathrm{MHz}$ ). It should be noted that metal particles do not heat in a microwave electric field, since the electric field within the particles is screened to almost zero and so is unable to drive the heating process.

Conversely, in the RF part of the spectrum, heating is a result of the conversion of magnetic energy, generally termed "induction heating", and can be initiated even in dielectric samples by the addition of small conducting or magnetic particles. Induction heating is driven by the eddy currents induced in the particles when conducting, or the magnetization currents when magnetic. The heat is then conduced by conventional means into the surrounding host material.

The advantages offered by microwaves are the rapid, uniform, and selective heating involving no direct contact between the microwave source and the heating material. The material to be heated can be thought of as simply being "bathed" in microwaves, with the resulting heat being generated volumetrically and almost uniformly throughout the material. Water-containing materials are most effectively heated around $20 \mathrm{GHz}$, but field penetration drops rapidly with increasing frequencies so that $2.45 \mathrm{GHz}$ is used (as a compromise) for domestic ovens, where penetration depths are then many centimeters.

High power microwaves (HPMW) are traditionally generated by valve-type sources (e.g., magnetrons), which are very efficient at converting electrical input power into microwave power (typically around $80 \%$ ). The allocated ISM (i.e. industrial, scientific and medical) frequencies for HPMW in the UK are 896 and 2,450 MHz. Continuous microwave powers of over $100 \mathrm{~kW}$ can be delivered at $896 \mathrm{MHz}$, increasing to over $1 \mathrm{MW}$ if the power is pulsed (even $>1 \mathrm{GW}$ for extremely short bursts) [5]. Generally, output power decreases with increasing frequency, but very high power gyrotron sources are now being reported even at frequencies of $30 \mathrm{GHz}$ and above [6].

Microwaves are currently utilized in many industrial heating applications such as food drying and processing
[7], chemical synthesis [8, 9], digestion [10, 11] and extraction [12-15]. Successful microwave pyrolysis studies have been conducted with a variety of feedstocks and resulting products (e.g., [16-20]). Two useful books that cover the application of microwaves in industrial heating applications are [21] and [22].

Microwave and RF heating methods have been used to recover oil and gas from oil shale and oil sands [15, 23, 24 ], but there have been only limited reports of microwaveassisted cracking of heavy oil hydrocarbons in the scientific literature. Two general reviews of the use of microwaves in the petrochemical industry are [25] and [26]. Early studies by Wan et al. used microwave-absorbing additives in reaction mixtures owing to the naturally low microwave losses of the associated organic materials, and described the microwave-assisted catalytic hydrogenation of alkenes [27, 28],. Fanson et al. [29] described the microwaveassisted cracking of hydrocarbons in the presence of a precious metal impregnated, metal oxide catalyst, with high conversion efficiency in the presence of carbon at atmospheric pressure. Tooley [30] describes an apparatus for the extreme microwave heating of liquids, with application for the cracking of waste oil. Recent studies [31] have demonstrated that even the moderate power provided by a domestic microwave oven is sufficient to improve the olefin yields from small volumes of crude oil without the addition of catalyst or absorbing additive. From this overview of the literature, we conclude that there are major opportunities for the use of microwave technologies in petroleum refinement.

\section{Background theory for efficient heating}

If an electric field is set up within a dielectric material, the interaction between the material and field is described by the material's relative permittivity $\varepsilon$. This links the polarization per unit volume $\underline{P}$ in terms of the internal electric field $\underline{E}_{\text {in }}$ via the relationship $\underline{P}=(\varepsilon-1) \varepsilon_{0} \underline{E}_{\text {in }}$. We consider losses by writing $\varepsilon$ as the complex quantity $\varepsilon=\varepsilon_{1}-j \varepsilon_{2}$, where $\varepsilon_{1}$ [or, more correctly the quantity $\left(\varepsilon_{1}-1\right)$ ] is proportional to the material's polarization and $\varepsilon_{2}$ the power dissipation, which we will consider in more detail below.

A resonant microwave cavity is an efficient structure for converting microwave energy into heat. These are robust metal structures that can withstand the rigors of an industrial environment that are variants of the common domestic oven, except that they are often designed for single mode operation. Very high conversion efficiency of microwave energy into heat is possible since the sample load is usually well known and characterized (unlike that for domestic ovens). Figure 1 shows schematically the simplified principles of a microwave actuator based on a cavity resonator 


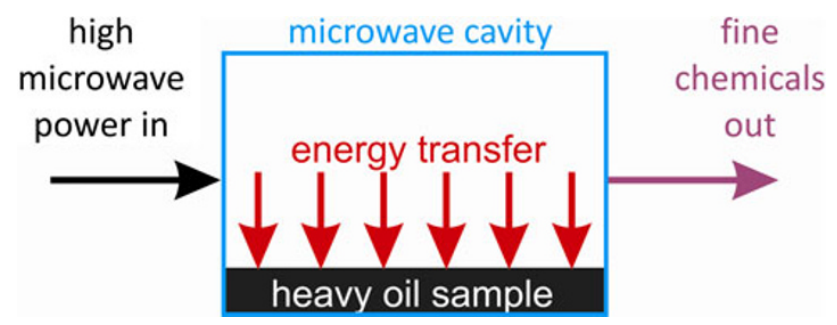

Fig. 1 A schematic of a HPMW heating system to process crude oil, based on a high power microwave cavity to enable efficient power conversion between the microwave electric field and heat in the sample. Diagnostic testing of the output (e.g., by mass spectrometry) can be used to detect for olefin generation

used to process crude oil. We will now look at the design considerations based on the dielectric properties of the material to be heated.

The unloaded quality factor of a resonant cavity is given by [32]

$Q=2 \pi f_{0} \frac{\text { average stored energy }}{\text { average power dissipated }}=2 \pi f_{0} \frac{U}{W}$

Where $f_{0}$ is the resonant frequency. The coupling structure between the microwave source and the cavity can usually be adjusted on a bespoke system so that no power is reflected and the cavity is impedance matched to the source. The cavity is then said to be critically coupled, there is no reflected power, and the average power dissipated within the cavity equals the input power $W_{\text {in }}$ from the source. This is clearly desirable for microwave heating applications, resulting in maximum conversion efficiency of electromagnetic energy into heat.

How much of this microwave input power ends up as heat in the sample? To answer this, we assume that the presence of the sample does not reconfigure the electromagnetic fields in the cavity to a major extent (i.e. it presents a small perturbation) and we use Eq. (1) to write

$W_{\text {in }}=W_{s}+W_{0}=2 \pi f_{0} \frac{U}{Q} \approx W_{0} \frac{Q_{0}}{Q}$

where $W_{\mathrm{s}}$ is the average power dissipated in the sample, $W_{0}$ is that dissipated in the cavity walls, $Q$ is the quality factor of the cavity when containing the sample and $Q_{0}$ is that when the cavity is empty. Rearranging Eq. (2), then gives us a very simple formula for the efficiency (in \%) of microwave energy conversion into sample heat

$\frac{W_{\mathrm{s}}}{W_{\text {in }}} \approx\left(1-\frac{Q}{Q_{0}}\right) \times 100 \%$

Hence, to design an efficient microwave cavity (i.e. actuator) for heating, we need to ensure that the introduction of the sample into the cavity reduces its $Q$ factor as much as possible. To illustrate the importance and usefulness of Eq. (3), if the sample reduces the $Q$ factor (a)

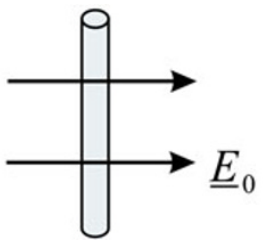

(b)

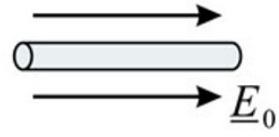

Fig. 2 Depolarization effects in dielectric cylinders. In case a, the magnitude of the internal electric field is reduced by a factor $2 /(\varepsilon+2)$ compared to the applied field $E_{0}$, resulting in a very small internal field when the relative permittivity $\varepsilon>>1$. In case $\mathbf{b}$, the continuity of the parallel component of $E$ at a boundary between two media ensures that the magnitude of the internal electric field is approximately equal to the applied field $E_{0}$

from 5,000 to 4,000 then the expected conversion efficiency when the cavity is critically coupled will be around $20 \%$, but if $Q$ is reduced from 5,000 to less than 500 then the conversion efficiency will be greater than $90 \%$.

To ensure the greatest impact of the sample on the $Q$ factor, we must design the actuator so that depolarization effects are kept to a minimum, thus maintaining a high electric field within the sample. The average power dissipated within a lossy dielectric is

$W=\frac{1}{2} \omega_{0} \varepsilon_{0} \varepsilon_{2} E_{\text {in }}^{2} V$

where $V$ is the sample volume, and $E_{\text {in }}$ is the magnitude of the internal electric field (here assumed to be uniform, which is a good general approximation). The development of polarization charges on dielectric surfaces perpendicular to the applied electric field $E_{0}$ means that $E_{\text {in }}<E_{0}$, which can give rise to a dramatic reduction in the heating rate in materials with large values of $\varepsilon_{1}$. This is shown schematically in Fig. 2, where we assume that a cylindrical liquid sample is introduced into a microwave cavity with an applied electric field $E_{0}$ either parallel or perpendicular to the axis of the cylinder. The ratio of internal electrical fields and power dissipation in the two cases (assuming the same applied field) are

$$
\begin{aligned}
& \frac{E_{\mathrm{in}}^{\|}}{E_{\mathrm{in}}^{\perp}} \approx \frac{1}{2}(\varepsilon+1) \approx \frac{1}{2}\left(\left(\varepsilon_{1}+1\right)-j \varepsilon_{2}\right), \\
& \frac{W_{\|}}{W_{\perp}} \approx \frac{1}{4}\left(\left(\varepsilon_{1}+1\right)^{2}+\varepsilon_{2}^{2}\right)
\end{aligned}
$$

respectively. For example, for water at $900 \mathrm{MHz}$ where $\varepsilon=80-j 3.4$, the power dissipation ratio estimated from Eq. (5) is greater than 1,600 . This means that it is hugely beneficial to design an actuator so that the electric field is parallel to the sample, which for liquids is most easily accomplished by drawing them into a thin cylinder. The same is true for oil samples but the effects of depolarization are not as dramatic as for water, since for oil $\varepsilon$ is much smaller. 
We next present our measurements of the dielectric properties of crude oil, from which we can infer the heating conversion rates within a given actuator structure.

\section{Experimental results}

Two heavy oil samples were measured, one Saudi heavy crude oil and one Saudi bunker oil, both supplied by Saudi Aramco. The physical properties of the oil samples are shown in Table 1. It is seen that the bunker oil has higher sulfur and metal content than the crude oil sample, while there are less aliphatics in the bunker oil.

The first method of microwave characterization was the broadband coaxial probe, which is a standard tool for measuring the complex permittivity of liquids [33]. Our probe is a miniaturized, bespoke probe based around an Anritsu K-connector (shown schematically in Fig. 3), which has to be carefully calibrated using the procedure outlined in [34]. Briefly, the $1.6 \mathrm{~mm}$ diameter aperture presented to the probe is very small for frequencies below $10 \mathrm{GHz}$ (where the free space wavelength is greater than $30 \mathrm{~mm}$ ). This allows us to use a simple capacitive model for extracting the complex permittivity $\varepsilon$ of the sample, so that the admittance of the aperture with and without the sample is $Y_{\mathrm{s}}=j \omega\left(\varepsilon C_{0}+C_{\mathrm{f}}\right)$ and $Y_{0}=j \omega\left(C_{0}+C_{\mathrm{f}}\right)$, where $C_{0}$ is the probe's air-spaced capacitance, $C_{\mathrm{f}}$ is the probe's fringing capacitance and $\omega$ is the (angular) frequency.

We extract experimental values of the probe admittance by measuring the voltage reflection coefficient $S_{11}$ with and without the sample (allowing us to calibrate out to the electrical length of any cables). The capacitance terms are found from measurements of the standard polar liquids methanol and ethanol, which can be obtained in very high purity and which have well-known values of complex permittivity (both well-described by the Debye model for polar liquids). A drop of each oil sample is placed over the aperture, fully covering it to a depth of about $2 \mathrm{~mm}$. All measurements were taken using an Agilent E5071B vector network analyzer (VNA) over the frequency range

Table 1 The physical properties of the oil samples used in this work

\begin{tabular}{lll}
\hline Index & Crude oil & Bunker oil \\
\hline Density $\left(\mathrm{kg} / \mathrm{m}^{3}\right)$ & 860 & 913 \\
API gravity & 27.0 & 23.4 \\
Sulfur (wt \%) & 2.2 & 3.4 \\
Total nitrogen content (wppm) & 1,300 & 1,600 \\
Total metal content (wppm) (V, Ni, Fe) & 120 & 187 \\
Aliphatics $(w t \%)$ & 37 & 27 \\
Pour point $\left({ }^{\circ} \mathrm{C}\right)$ & 33.5 & 38.9 \\
Carbon residue, RAMS (wt \%) & 7.1 & 8.5 \\
\hline
\end{tabular}

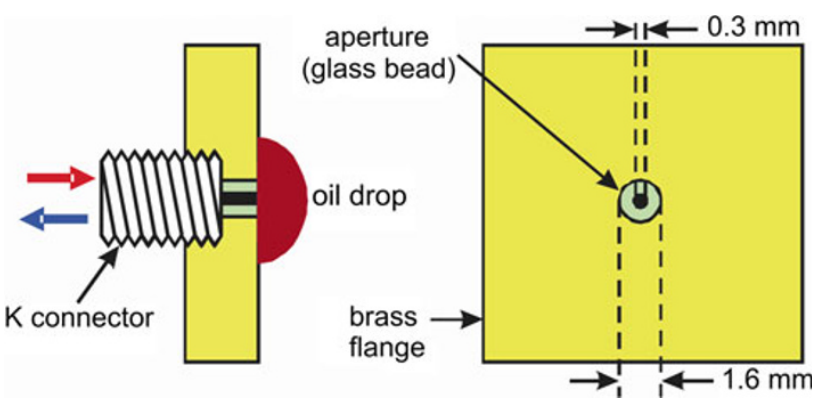

Fig. 3 A schematic diagram of the coaxial reflectance probe used to determine the broadband $(0.1-8 \mathrm{GHz})$ complex permittivity $\varepsilon$ of a sample that covers the aperture

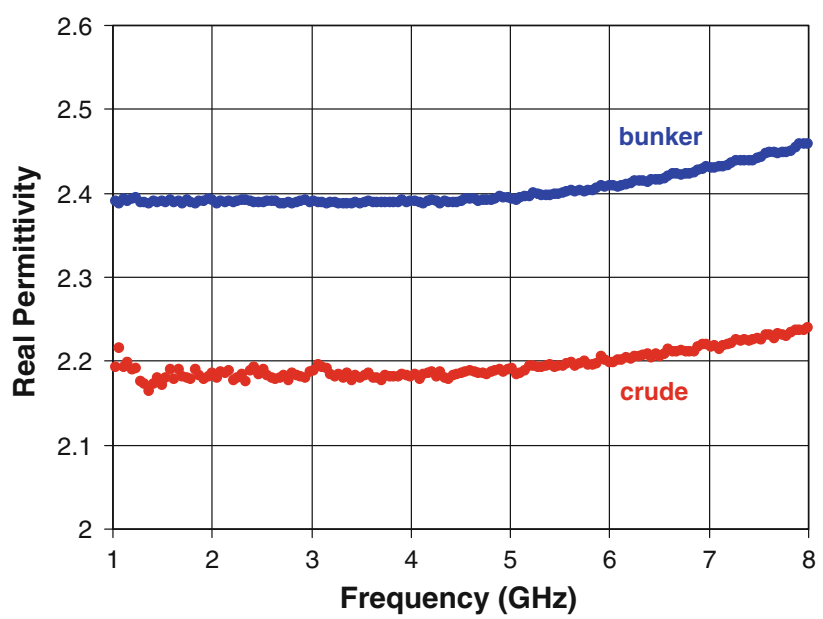

Fig. 4 The real part $\varepsilon_{1}$ of the complex permittivity of two heavy oil samples measured with the broadband coaxial probe

$10-8.5 \mathrm{GHz}$ and the data are read into a Mathcad program that performs the necessary computations to extract $\varepsilon$.

Data for the real part of complex permittivity $\varepsilon_{1}$ are shown in Fig. 4 for both heavy oil samples. The values vary little with frequency and remain in the range of 2.2-2.5 over the full bandwidth of the measurement, indicating low inherent electrical polarization. The imaginary (i.e. lossy) part of the complex permittivity $\varepsilon_{2}$ is shown in Fig. 5. Again, the values are small, consistent with the low polarization, and increase roughly quadratically with increasing frequency. At industrially relevant frequencies, the complex permittivities are found to be for crude oil $\varepsilon=2.19-j 0.003$ at $900 \mathrm{MHz}$ and $\varepsilon=2.19-j 0.007$ at $2.45 \mathrm{GHz}$; for bunker oil $\varepsilon=2.39-j 0.002$ at $900 \mathrm{MHz}$ and $\varepsilon=2.39-j 0.005$ at $2.45 \mathrm{GHz}$.

Referring to the physical data of Table 1, the fact that bunker oil has a slightly higher permittivity (i.e. $\varepsilon_{1}$ ) than crude oil over the full frequency range could be due to its higher mass density (linked also to its lower aliphatic content). The lower loss $\varepsilon_{2}$ of bunker oil (by around a factor of 2 compared to crude oil) is most likely due to a 


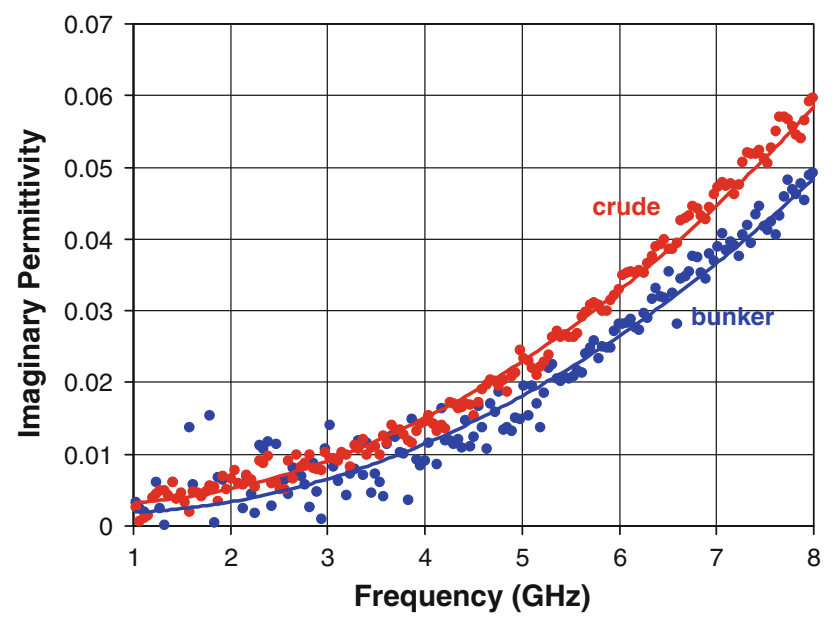

Fig. 5 The imaginary part $\varepsilon_{2}$ of the complex permittivity of two heavy oil samples again measured using the broadband coaxial probe

lower concentration of polar species, but this is not linked inherently to any of the properties of Table 1 .

To obtain more accurate experimental values for the loss $\varepsilon_{2}$, and hence to assess the heating conversion efficiency of these samples when placed in a suitably designed microwave cavity, the measurements were repeated in a $\mathrm{TM}_{010}$ cylindrical cavity operating at $2.45 \mathrm{GHz}$. The cavity is made from two D-shaped cups machined from solid aluminum, with inner cavity radius of $4.6 \mathrm{~cm}$ and inner cavity length of $4.0 \mathrm{~cm}$. The samples were placed in polythene cylindrical tubes of inner diameter $4.5 \mathrm{~mm}$, of negligible dielectric loss, running the full internal length of the cavity. A schematic of the cavity set-up is shown in Fig. 6, where it can be seen that the applied electric field is parallel to the sample, giving the maximum possible reduction in $Q$ factor (i.e. the maximum possible electric field coupling to the sample). The cavity's microwave magnetic field is excited with a pair of identical coaxial SMA launchers terminated in short-circuit loops and the transmitted power is measured with the same VNA as for the coaxial probe measurements. Typical measurement results are shown in Fig. 7.

The complex permittivity of the two heavy oil samples were calculated using first-order cavity perturbation theory [35] from the experimentally observed changes in resonant frequency $f_{0}$ and $Q$ factor, using

$\frac{\Delta f_{0}}{f_{0}} \approx-\left(\varepsilon_{1}-1\right) \frac{V_{\mathrm{s}}}{2 V_{\mathrm{eff}}}$

$\Delta\left(\frac{1}{2 Q}\right) \approx \varepsilon_{2} \frac{V_{\mathrm{s}}}{2 V_{\mathrm{eff}}}$

where $V_{\mathrm{s}}$ is the volume of sample within the cavity (i.e. $0.64 \mathrm{~cm}^{3}$ ) and $V_{\text {eff }}$ is the effective volume of the cavity, which is found by calibration measurements of a range of metal spheres to be $69.4 \pm 0.1 \mathrm{~cm}^{3}$ (this compares well with (a)

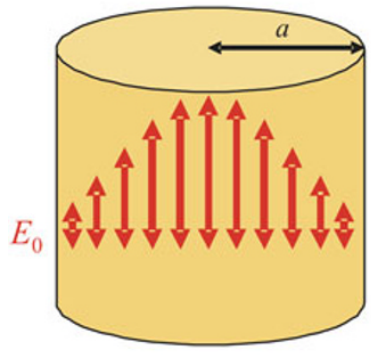

(b)

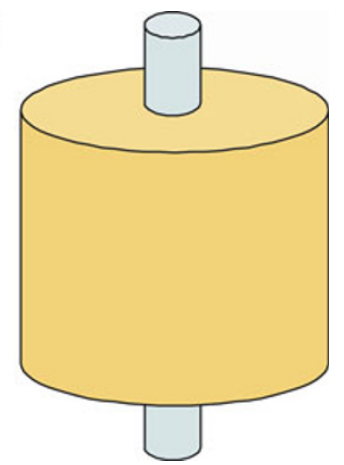

Fig. 6 a A schematic diagram of the $\mathrm{TM}_{010}$ cylindrical cavity and its electric field distribution, used to measure the complex permittivity of oil samples around $2.45 \mathrm{GHz}$. In terms of the internal radius $a$ (in $\mathrm{cm}$ ), the resonant frequency (in $\mathrm{GHz}$ ) is given by $f_{0}=11.5 / a$ (e.g., an internal radius of $4.6 \mathrm{~cm}$ gives a resonant frequency of $2.5 \mathrm{GHz}$ ). b A liquid sample (e.g., oil) is inserted along the cavity's axis, contained within a low-loss tube. This parallel field configuration minimizes the effects of sample depolarization and provides the strongest coupling between the cavity and sample. Here, the cavity is coupled via its magnetic field (not shown) by a pair of loop-terminated coaxial cables

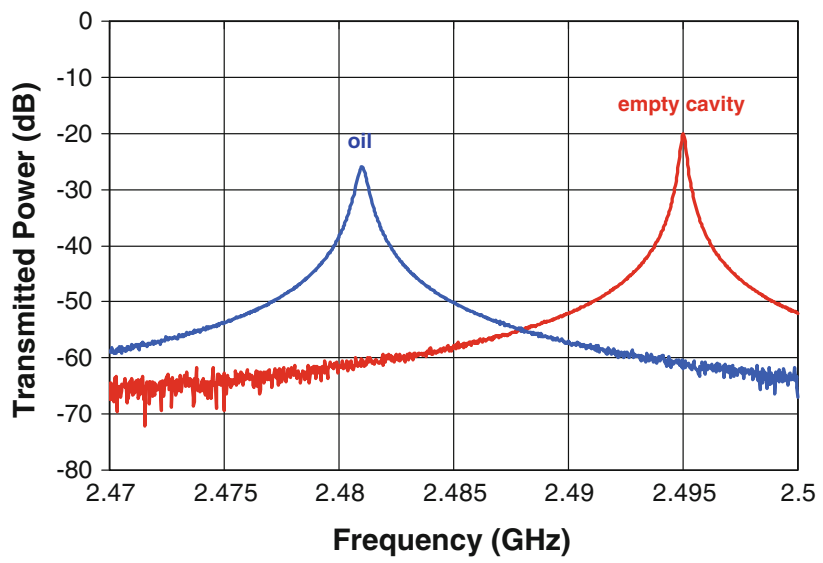

Fig. 7 The $\mathrm{TM}_{010}$ cavity response with and without a crude oil sample. There is very little change in the resonant bandwidth, indicative of low dielectric loss

the theoretically predicated value of $71.7 \mathrm{~cm}^{3}$ ). At the resonant frequency of $2.48 \mathrm{GHz}$, the complex permittivity values of crude and bunker oil are $\varepsilon=2.20-j 0.0071$ and $\varepsilon=2.38-j 0.0048$, respectively, with $\pm 2 \%$ measurement error in both real and imaginary parts of $\varepsilon$. These cavity data are in excellent agreement with the coaxial probe data at the same frequency, and give us confidence to predict the conversion efficiency if these samples were to be placed in a suitable microwave actuator.

\section{Discussion}

In this paper, we have reviewed the use of high power microwaves and presented some of our preliminary results 

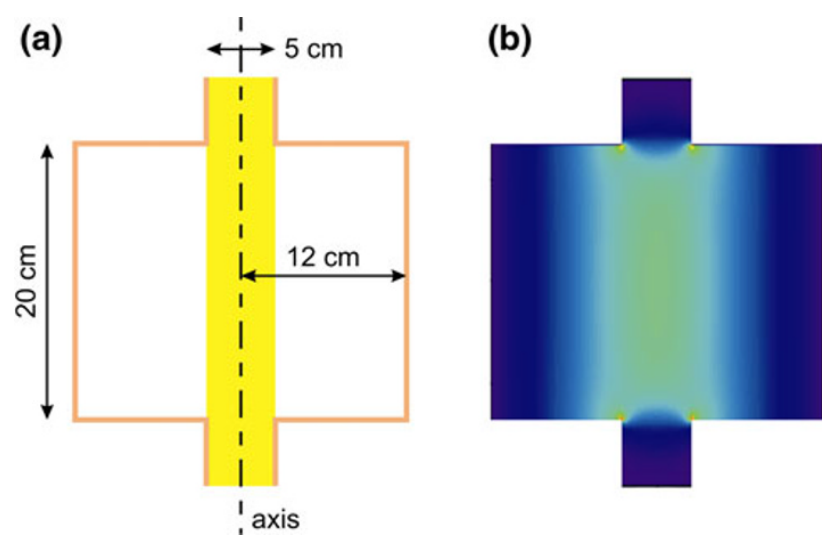

Fig. 8 a Schematic diagram of a microwave heater based on a copper $\mathrm{TM}_{010}$ cavity operating around $900 \mathrm{MHz}$ (inner cavity radius of $12 \mathrm{~cm}$ and a length of $20 \mathrm{~cm}$ ), with the crude oil column shown. b The simulated distribution of the resulting electric field magnitude, which drives the heating. The entry and exit holes for the oil each have radii of $2.5 \mathrm{~cm}$, so form cylindrical waveguides which are well below their cut-off frequency. This means that the microwave fields are contained within the cylindrical cavity space and are concentrated along the axial region, where the electric field is large, approximately uniform, and directed parallel to the axis

for the microwave characterization of crude and bunker oil samples, using both a broadband coaxial probe and highly sensitive $\mathrm{TM}_{010}$ cavity working around $2.45 \mathrm{GHz}$. Finally, we now discuss how we can use the measured values of complex permittivity $\varepsilon$ to calculate the heating efficiency in the raw heavy oils, initially without additives, in a suitable microwave actuator.

We will work around $900 \mathrm{MHz}$ since then a single mode $\left(\mathrm{TM}_{010}\right)$ cavity actuator has an internal radius of around $12 \mathrm{~cm}$ and so can accommodate larger liquid volumes. A suitable design is shown in Fig. 8a, where the length of the cavity $(20 \mathrm{~cm})$ is chosen to be long enough to give it a high $Q$ factor when empty (around 32,000 if made from copper, or when copper coated), but short enough to ensure that higher order cavity modes (e.g., $\mathrm{TE}_{111}$ ) do not interfere with the fundamental $\mathrm{TM}_{010}$ mode. Here we envisage a flow of oil through a low-loss tube, with the cylindrical inlet and outlet holes shown. We have assumed these to be of internal diameter of $5 \mathrm{~cm}$, meaning that they act as waveguides well below their lowest cut-off frequency so do not affect the distribution of electromagnetic field within the cavity, or cause radiative losses which may otherwise be a safety hazard when using very high power microwave sources.

We have simulated the fields in the cavity using Poisson Superfish [36], which can used for calculating the electromagnetic fields in cylindrical cavities for axially symmetric modes (as is the case for $\mathrm{TM}_{010}$ ). We use the complex permittivity $\varepsilon=2.19-j 0.005$ for crude oil at $900 \mathrm{MHz}$ taken from the coaxial probe data of Figs. 4 and 5. The resulting electric field magnitude is shown in

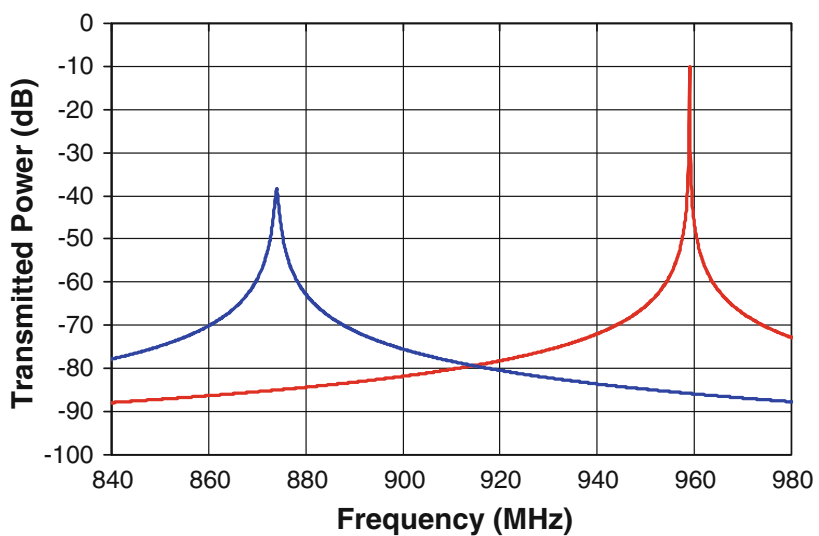

Fig. 9 The simulated response of the cavity shown in Fig. 8. Based on the measured microwave properties of crude oil at $900 \mathrm{MHz}$, if the oil forms an axial liquid column of radius $2.5 \mathrm{~cm}$, the cavity $Q$ factor drops from 32,000 when empty (if made from copper), to 1,200 when bunker oil is present (left hand trace). If the cavity is critically coupled to its high power microwave source, this means that $96 \%$ of the input microwave power is converted to heat in the oil, with the remaining $4 \%$ appearing as heat in the cavity walls

Fig. 8b, which has the required property of being concentrated in the region where the oil sample resides. In addition, as with the earlier cavity perturbation experiments, the direction of $E$ is parallel to the axis of the oil cylinder, thus giving the largest possible value of the internal electric field within the oil to drive the heating.

From these fields, we calculate that the $Q$ factor of the cavity is reduced from 32,000 to 1,200 due to the presence of the raw crude oil, and from Eq. (3) we estimate a heating conversion efficiency for about $96 \%$. The simulated spectral response of the cavity with and without the crude oil sample is shown in Fig. 9. Assuming the cavity is critically coupled, this means that $100 \mathrm{~kW}$ of incident microwave power leads to about $96 \mathrm{~kW}$ being generated as heat within the sample, with the remainder being dissipated as heat in the cavity walls (which may have to be aircooled). On the one hand, this conversion efficiency is encouragingly high, but on the other hand the volume of oil needs to be large (here $\pi \times 2.5 \mathrm{~cm} \times 2.5 \mathrm{~cm} \times 20 \mathrm{~cm}$ $\approx 0.4 \mathrm{~L}$ ) since its microwave loss is so small. Clearly, the high resulting heat capacity due to the large oil mass will limit the heating rate of the oil. To reduce the oil volume whilst preserving similar ( $>95 \%)$ conversion efficiencies, the value of $\varepsilon_{2}$ can be increased dramatically by the addition of a microwave absorbing phase.

To compare the heating efficiency of different samples in a $\mathrm{TM}_{010}$ cavity, a useful figure of merit is the product $\varepsilon_{2}^{\text {eff }} V_{\mathrm{s}}$, which we aim to get as high as possible. Here $V_{\mathrm{s}}$ is the sample volume and $\varepsilon_{2}^{\text {eff }}$ is the effective loss of the oil emulsion including the absorbing phase, which depends mostly on $\varepsilon_{2}$ of the lossy phase and its volume fraction. Carbon blacks (and related materials) are ideal lossy 
additives, with well-known high values of microwave absorption (e.g., $\varepsilon_{2}$ in the range of 1-10 for carbon black composites at microwave frequencies [37]). Hence, we can get $>95 \%$ conversion efficiency for a carbon black inclusion volume of only about $0.2 \mathrm{~mL}$, meaning that the oil volume can be scaled down to 10 s of $\mathrm{mL}$, thus reducing its heat capacity by an equivalent ratio to the volume reduction.

\section{Conclusions}

To conclude, we have determined the complex permittivity of heavy oil samples at microwave frequencies. We have then used these results to model the efficiency of heat generation in a flow system suitable for microwave-assisted cracking. Whilst the microwave loss of even heavy oils is found to be small, it is possible to have very high microwave conversion efficiencies for moderate volumes of liquid. If the thermal mass is too large, high conversion efficiency can be maintained for smaller volumes by addition of a highly absorbing additive. In the future, we will perform experiments on such oil emulsions to characterize their microwave properties, and build an efficient desktop system to evaluate the fundamental principles associated with the microwave-assisted cracking of heavy oils.

Acknowledgments We thank Merck KGaA for the sponsorship of Dan Slocombe.

Open Access This article is distributed under the terms of the Creative Commons Attribution License which permits any use, distribution, and reproduction in any medium, provided the original author(s) and the source are credited.

\section{References}

1. Mingos DMP, Baghurst DR (1991) Applications of microwave dielectric heating effects to synthetic problems in chemistry. Chem Soc Rev 20:1-47

2. Zhang X, Hayard DO, Mingos DMP (2003) Effects of microwave dielectric heating on heterogeneous catalysis. Catal Lett 88:33-38

3. Jones DA, Lelyveld TP, Mavrofidis SD, Kingman SW, Miles NJ (2002) Microwave heating applications in environmental engineering-a review. Resour Conserv Recycl 34(2002):75-90

4. Thostenson ET, Chou TW (1999) Microwave processing: fundamentals and applications. Compos A 30:1055-1071

5. Gold SH, Nusinovich GS (1997) Review of high-power microwave source research. Rev Sci Instrum 68:3945-3974

6. Kumar N, Singh U, Kumar A, Khatun H, Singh TP, Sinha AK (2011) Design of $35 \mathrm{GHz}$ gyrotron for material processing applications. Prog Electromagn Res B 27:273-288

7. Venkatesh MS, Raghavan GSV (2004) An overview of microwave processing and dielectric properties of agri-food materials. Biosyst Eng 88:1-18

8. Kappe CO (2004) Controlled microwave heating in modern organic synthesis. Angew Chem Int Ed 43:6250-6284
9. Tian ZQ, Jiang SP, Liang YM, Shen PK (2006) Synthesis and characterization of platinum catalysts on multi-walled carbon nanotubes by intermittent microwave irradiation for fuel cell applications. J Phys Chem B 110:5343-5350

10. Chen CM, Chen M, Leu FC, Hsu SY, Wang SC, Shi SC, Chen CF (2004) Purification of multi-walled carbon nanotubes by microwave digestion method. Diam Relat Mater 13:1182-1186

11. Afridi HI, Kazi TG, Jamali MK, Kazi GH, Arain MB, Jalbani N, Shar GQ (2006) Analysis of heavy metals in scalp hair samples of hypertensive patients by conventional and microwave digestion methods. Spectrosc Lett. 39:203-214

12. Al-Harahsheh M, Kingman S, Somerfield C, Ababneh F (2009) Microwave-assisted total digestion of sulphide ores for multielement analysis. Anal Chim Acta 638:101-105

13. El Harfi K, Mokhlisse A, Chanaa MB, Outzourhit A (2000) Pyrolysis of the Moroccan (Tarfaya) oil shales under microwave irradiation. Fuel 79:733-742

14. Lucchesi ME, Chemat F, Smadja J (1043) Solvent-free microwave extraction of essential oil from aromatic herbs: comparison with conventional hydrodistillation. J Chromatogr A 2004: 323-327

15. Robinson JP, Kingman SW, Onobrakpeya O (2008) Microwaveassisted stripping of oil contaminated drill cuttings. J Environ Manag 88:211-218

16. Ludlow-Palafox C, Chase HA (2001) Microwave-induced pyrolysis of plastic wastes. Ind Eng Chem Res 40:4749-4756

17. Menendez JA, Inguanzo M, Pis JJ (2002) Microwave-induced pyrolysis of sewage sludge. Water Res 36:3261-3264

18. Huang YF, Kuan WH, Lo SL, Lin CF (2008) Total recovery of resources and energy from rice straw using microwave-induced pyrolysis. Bioresour Technol 99:8252-8258

19. Yagmur E, Ozmak M, Aktas Z (2008) A novel method for production of activated carbon from waste tea by chemical activation with microwave energy. Fuel 87:3278-3285

20. Lei HW, Ren SJ, Julson J (2009) The effects of reaction temperature and time and particle size of corn stover on microwave pyrolysis. Energy Fuels 23:3254-3261

21. Metaxas AC, Meredith RJ (1981) IEE Power Engineering Series 4. Peter Peregrinus Ltd., London

22. Roussy G, Pearce JA (1996) Foundations and industrial applications of microwave and radio frequency fields. Wiley, New York

23. Pierre EP (1992) Microwave separation of bituminous material from tar sands. Canadian Patent 1293943

24. Bosisio RG, Cambon JL, Chavarie C, Klvana D (1977) Experimental results on the heating of Athabasca tar sand samples with microwave power. J Microw Power 12:301-307

25. Mutyala S, Fairbridge C, Paré JRJ, Bélanger JMR, Ng S, Hawkins R (2010) Microwave applications to oil sands and petroleum: a review. Fuel Process Technol 91:127-135

26. Kustov LM, Sinev IM (2010) Microwave activation of catalysts and catalytic processes. Russ J Phys Chem A 84:1676-1694

27. Wan JKS, Kriz JF (1985) Hydrodesulphurization of hydro cracked pitch. US patent 4545879

28. Wan JKS, Wolf K, Heyding RD (1984) Some chemical aspects of microwave assisted catalytic hydro-assisted processes. In: Kaliaguine S, Mahay A (eds) Catalysis on the energy scene. Elsevier, Amsterdam

29. Fanson PT, Hirato H, Masaya IBE, Suib SL, Son YC (2006) Process using microwave energy and a catalyst to crack hydrocarbons. PCT International Patent, WO/2006/083829

30. Tooley J (2008) Process for cracking of waste oil by microwave. US Patent 2008/0202982 A1

31. Mohammed AD, Mohammed IA, Ajinomoh CS (2011) Upgrading heavy crude oil potentials through microwave assisted distillation. J Innov Res Eng Sci 2:137-147 
32. Pozar DM (2011) Microwave engineering. Wiley, New York

33. McLaughlin BL, Robertson PA (2007) Miniature open-ended coaxial probes for dielectric spectroscopy applications. J Phys D Appl Phys 40:45-53

34. Sulaimalebbe A, Porch A, Vidal-Iglesias FJ, Attard G (2008) Microwave properties of platinum nanoparticle films. In: IEEE MTT-S International Microwave Symposium Digest, pp 1585-1588

35. Shi LH, Akyel C, Bosisio RG (1981) Precise calculations and measurements on the complex dielectric constant of lossy materials using TM010 cavity perturbation techniques. IEEE Trans Microw Theory Tech. 29:1041-1048

36. Poisson Superfish, Los Alamos National Laboratory. http:// laacg1.lanl.gov/laacg/services

37. Hotta M, Hayashi M, Lanagan MT, Agrawal DK, Nagata K (2011) Complex permittivity of graphite, carbon black and coal powders in the ranges of $\mathrm{X}$-band frequencies $(8.2$ to $12.4 \mathrm{GHz})$ and between 1 and $10 \mathrm{GHz}$. ISIJ Int 51:1766-1772 